\title{
Impaired recovery of hind limb muscle perfusion following ischemic injury in rodents receiving $B$ vitamin supplementation
}

Paul J Lee $^{1,2}$, Sarah Desjardins ${ }^{1}$, Kevin Sorokin ${ }^{1}$, Louisa Ho ${ }^{1}$, Christine Liao ${ }^{2}$, Michael Kuliszewski ${ }^{2}$, James D House ${ }^{3}$, Nicole Yuen ${ }^{1,4}$, Parastoo Azizi Namini ${ }^{4}$, Mavra Ahmed ${ }^{4}$, Howard Leong-Poi ${ }^{2,5}$ and Mary Keith ${ }^{1,4 *}$

${ }^{1}$ Division of Nutrition, Keenan Research Centre in the Li Ka Shing Knowledge Institute, St. Michael's Hospital, Canada

${ }^{2}$ Division of Cardiology, St. Michael's Hospital, Toronto, Ontario, Canada

${ }^{3}$ Department of Human Nutritional Sciences, University of Manitoba, Winnipeg, Manitoba, Canada

${ }^{4}$ Department of Nutritional Sciences, University of Toronto, Toronto, Ontario, Canada

${ }^{5}$ Department of Medicine, University of Toronto, Toronto, Ontario, Canada

\begin{abstract}
Background: B vitamin deficiency has been previously reported to be prevalent in patients with heart failure. However, clinical trials investigating the potential of B vitamin supplementation have failed to show benefit and have suggested some evidence of harm. Negative findings have been hypothesized to be the result of an anti-angiogenic effect associated with B vitamin supplementation.
\end{abstract}

Objective: The current study aimed to determine the impact of vitamin B6 supplementation alone or in combination with folate and B12 on the angiogenic response in a rodent model of hind limb ischemia.

Methods: Rats were divided randomly into three groups and fed one of three diets: control diet (CON), high B6 diet (HB6) or high B6+folate+B12 diet (TV). Following five weeks of diet therapy, ischemia was surgically induced in the hind limb. Changes in perfusion and markers of the angiogenic response were studied at either 5 (early) or 10 (late) weeks.

Result: Circulating EPCs were reduced in HB6 animals, a finding that reached significance for the TV animals. Quantitative polymerase chain reaction analysis revealed a decline in VEGF and eNOS expression in HB6 and TV groups at week 10. Contrast enhanced ultrasound of the hind limb revealed a significant reduction in perfusion in HB6 and TV animals in comparison to CON at 10 weeks. B vitamin supplementation had no impact on EPC apoptosis or differentiation.

Conclusion: Taken together, these results support a negative impact of B vitamin supplementation on the recovery of perfusion following ischemic injury which may be the result of a down-regulation of chemotactic gene expression, leading to lowered EPC recruitment from the bone marrow to the site of ischemic injury.

Abbreviations: cDNA: Complementary Deoxyribonucleic Acid; CEU: Contrast Enhanced Ultrasound; CON: Control; CVD: Cardiovascular Disease; eNOS: Endothelial Nitric Oxide Synthase; EPC: Endothelial Progenitor Cell; HB6: high B6 diet; HCy: Homocysteine; HOPE-2: Heart Outcomes Prevention Evaluation -2 Trial; LDPI: Laser Doppler Perfusion Imaging; PLP: Pyridoxal 5'-phosphate; qPCR: Quantitative Polymerase Chain Reaction;

RBC: Red Blood Cell; RNA: Ribonucleic Acid; TV: Tri vitamin diet (high B6, B12, Folic Acid); TUNEL: Terminal deoxynucleotidyl transferase dUTP nick end labelling; VEGF: Vascular Endothelial Growth Factor

\section{Introduction}

B vitamins play a vital role as cofactors in cellular energy metabolism, red blood cell formation and homocysteine (HCy) metabolism. Of particular importance is the role of vitamin B6 in HCy metabolism. Vitamin B6 refers to a group of chemically similar and interconvertible compounds, the active form being pyridoxal 5'-phosphate (PLP).
Increased concentrations of plasma $\mathrm{HCy}$ have been implicated as a predictor of vascular disease. As a result, there has been significant interest in the potential of $\mathrm{B}$ vitamins to both reduce circulating levels of HCy and subsequently cardiovascular disease (CVD) risk. However, two large randomized clinical trials in patients with previous established heart disease did not find any benefit to taking B vitamin supplements despite a reduction in homocysteine levels in the blood [1,2]. Furthermore, the Norwegian Vitamin Trial (NORVIT), a large vitamin supplementation trial in patients immediately following

Correspondence to: Mary Keith, Niagara North Family Health Team, 145 Carlton Street Unit \#7, St. Catharines, Ontario, Canada; E-mail: mkeith@ niagaranorthfht.ca

Key words: Complementary Deoxyribonucleic Acid; Endothelial Nitric Oxide Synthase; Endothelial Progenitor Cell

Received: September 20, 2017; Accepted: October 13, 2017; Published: October 16,2017 
myocardial infarction, found that vitamin B6, in combination with folic acid and B12 supplementation, resulted in a significant increase in adverse cardiac events, despite a decrease in plasma homocysteine levels, suggesting a potential adverse effect of vitamin supplementation in this population [3]. One explanation for these seemingly conflicting findings lies in the potential of B vitamin supplements to exert an antiangiogenic effect in vivo. This hypothesis is supported by animal studies in which B6 supplementation led to decreased blood vessel formation and subsequently impaired tumor growth [4-6].

Data from our laboratory suggests that vitamin B deficiency in patients with heart failure is prevalent and in the range of $27-38 \%$ depending upon the $\mathrm{B}$ vitamin examined $[7,8]$. As a result, $\mathrm{B}$ vitamin supplementation may be considered as a safe and potentially beneficial adjunct therapy in clinical practice. However, in light of the findings of the NORVIT trial and the possible negative impact of B vitamin supplementation, it is important to clarify the impact of B vitamin supplementation in a population where ischemia may be present and for whom the angiogenic response is critical. Therefore, we have completed a series of studies aimed at evaluating the early and late impact of B6 supplementation alone or in combination with B12 and folate on the angiogenic response in vivo. We hypothesized that in a rodent model of chronic limb ischemia, B6-enriched diets would retard the functional improvements associated with the angiogenic response including declined function of endothelial progenitor cells (EPCs). Furthermore, we hypothesized that the anti-angiogenic effect observed with B6 supplementation would be accentuated in the presence of B12 and folate, as observed in the NORVIT trial [3].

\section{Materials and methods}

\section{Animal protocol and experimental plan}

A total of 131 male Sprague Dawley rats (Charles River Laboratories, Montreal, Canada) of approximately 15 weeks of age (280-300g) were housed in accordance with the guidelines for the Care and Use of Laboratory Animals (Canadian Council on Animal Care, volume 1, 1993) and with the approval of the Animal Care Committee at the Keenan Research Centre of Li Ka Shing Knowledge Institute. At arrival, animals were randomized to one of three dietary treatments (Table 1):

\section{Control diet $(\mathrm{CON} ; \mathrm{N}=48)$}

2. High B6 supplementation ( $\mathrm{HB} 6 ; \mathrm{N}=47)$

\section{Trivitamin supplementation ( $\mathrm{TV} ; \mathrm{N}=46)$}

The CON group received the current recommended level of vitamin supplementation found in commercial rodent diets $(7 \mathrm{mg} / \mathrm{kg}$ of vitamin B6) [9] while the HB6 received $35 \mathrm{mg} / \mathrm{kg}$ diet of vitamin B6, and TV group received $35 \mathrm{mg} / \mathrm{kg}$ diet of vitamin B6, $358 \mu \mathrm{g} / \mathrm{kg}$ diet of vitamin B12, and $3.6 \mathrm{mg} / \mathrm{kg}$ diet of folate. Rodent housing was changed two times a week and food intake was quantified by weighing the leftover food pellets as well as carefully collecting any food pellets present in the bedding. All animals received food and water ad libitum and were maintained in a 12:12 hour light/dark cycle. Animal weight was monitored on a weekly basis.

Animals were sacrificed by exsanguination at 35 days post-surgery (10 weeks of diet treatment), with a subset of animals sacrificed at 3 days post-surgery ( 5 weeks of treatment) (Figure 1). Animals sacrificed at 5 weeks provided data both on the impact of dietary treatment alone as well as on any early post-ischemic effects of these modified diets. Hind limb tissue was harvested at time of sacrifice, and stored at $-80^{\circ}$ Celsius for gene expression analysis. Bone marrow-derived EPCs were isolated for quantification and functional assessment. A subset of animals sacrificed at the late (10 week) time point underwent fluorescent microangiography to visualize blood vessel formation.

\section{B Vitamin administration}

Rodent diets of defined nutrient composition were specially formulated by Dyets Inc (Bethlehem, Pennysylvania). All diets used

Table 1. Vitamin and Mineral Content of Defined Diets

\begin{tabular}{|c|c|c|c|c|c|}
\hline & $\begin{array}{c}117037 \\
\text { grams/kg } \\
7 \mathrm{mg} / \mathrm{kg} \mathrm{B6}\end{array}$ & $\begin{array}{c}117028 \text { High B6 } \\
\text { grams/kg } \\
35 \mathrm{mg} / \mathrm{kg} \mathrm{B6}\end{array}$ & $\begin{array}{c}117038 \\
\text { grams } / \mathbf{k g} \\
\text { Tri-Vitamin } \\
\text { B6+B12 + folic acid }\end{array}$ & $\begin{array}{l}\text { Dyet \#317004 } \\
\text { Vitamin Mix }\end{array}$ & $\begin{array}{c}\text { AIN-93 VX } \\
\text { Gram/kg }\end{array}$ \\
\hline Vitamin Free Casein & 200 & 200 & 200 & Niacin & 3 \\
\hline Cornstarch & 397.486 & 397.486 & 395.556 & Calcium Pantothenate & 1.6 \\
\hline Dextrose & 132 & 132 & 132 & Thiamin HCL & 0.6 \\
\hline Sucrose & 93 & 65 & 65 & Riboflavin & 0.6 \\
\hline Cellulose & 50 & 50 & 50 & Folic Acid & 0.2 \\
\hline Soybean Oil & 70 & 70 & 70 & Biotin & 0.02 \\
\hline tBHQ & 0.014 & 0.014 & 0.014 & Vitamin E Acetate $500 \mathrm{IU} / \mathrm{g}$ & 15 \\
\hline Mineral Mix \#210025 & 35 & 35 & 35 & Biotin & 0.02 \\
\hline Vitamin Mix \#317004 & 10 & 10 & 10 & Vitamin B12 (0.1\%) & 2.5 \\
\hline L-cystine & 3 & 3 & 3 & $\begin{array}{l}\text { Vitamin A Palmitate } \\
500000 \mathrm{IU} / \mathrm{g}\end{array}$ & 0.8 \\
\hline Choline Bitartrate & 2.5 & 2.5 & 2.5 & Vitamin D3 $400000 \mathrm{IU} / \mathrm{g}$ & 0.25 \\
\hline $\begin{array}{l}\text { Vitamin B12 premix (1mg/ } \\
\text { gm) }\end{array}$ & 0 & 0 & 35 & $\begin{array}{c}\text { Vitamin K1/Dextrose Mix } \\
(10 \mathrm{mg} / \mathrm{g})\end{array}$ & 7.5 \\
\hline Folic Acid Premix (1 mg/g) & 0 & 0 & 1.6 & Sucrose & 967.93 \\
\hline $\begin{array}{c}\text { Pyridoxine Premix } \\
1 \mathrm{mg} / \mathrm{gm}\end{array}$ & 7 & 35 & 35 & Total & 1000 \\
\hline Vitamin B12 (0.1\%) & 0 & 0 & 0.333 & & \\
\hline
\end{tabular}

Journal of Nutrition v123, 1941(1993)

CON: Control

HB6: High vitamin B6

TV: Tri-vitamin Group 


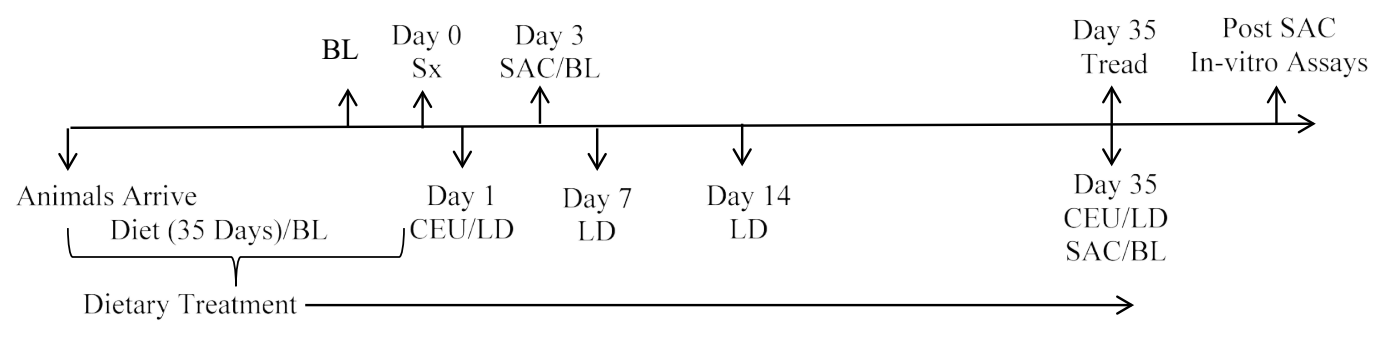

Figure 1. Study Design

Sx - Hindlimb Ischemia Surgery; CEU- Contrast Enhanced Ultrasound; LD - Laser Doppler Perfusion Imaging; Tread - Final Treadmill; SAC - Sacrifice; BL- B vitamin blood assessment

the standard AIN-93G [9] base with AIN-93-VX vitamin mix without pyridoxine thereby allowing for specific manipulation of the $B$ vitamin content, while maintaining identical concentrations of all macro- and micronutrients (Table 1). These diets were formulated to meet current rodent dietary recommendations for all nutrients with modifications to either vitamin B6 content alone (High B6) or enhanced B6, folate and B12 (Tri-vitamin Supplementation), as indicated by the study design [10]. The $35 \mathrm{mg} / \mathrm{kg}$ dose of vitamin B6 is based on previous studies demonstrating an anti-angiogenic effect of B6 on colon tumorigenesis $[4,5]$ and also reflects similar levels (on a per kg/body weight level) of B6 supplementation that might be consumed by an individual taking B complex vitamins $(2 \times 50 \mathrm{mg} / \mathrm{d}$ B complex vitamins $/ 70 \mathrm{~kg}$ adult). The TV group received the pharmacologic doses of B12 and folate adapted from trials investigating the effect of folate on endothelial function [11-13].

\section{Induction of ischemia in rats}

Following five weeks of diet treatment, animals were anesthetized with inhaled isofluorane. Using an aseptic technique, unilateral ischemia was achieved by the ligation of the left common iliac artery and its proximal branches. The ligation of proximal branches was done to ensure all blood supply to the hind limb muscle was ligated, as the adductor muscles receive blood from both the femoral artery and the pelvic plexus. Ligation was performed blinded to animal treatment and all animals were ligated on the same day in random order. This model of ischemia has been well established resulting in an immediate reduction (approximately $70 \%$ reduction in blood flow) in the ischemic limb compared to the contralateral non-ischemic limb and increases over the next 1-2 weeks post ligation, as measured by laser Doppler and contrast enhanced ultrasound, peaking and reaching a plateau at five weeks post-ligation [14].

\section{Laser doppler perfusion imaging (LDPI)}

The degree of blood vessel collateralization was investigated in the animals on days 1, 7, 14 and 34 days post-surgery. One day prior to laser Doppler analysis, the hair on the hind limb was completely removed using a commercial hair remover product and an electric shaver. Rodents were anesthetized with inhaled isofluorane, and underwent serial non-invasive measures of microvascular perfusion using the LDPI system (Moor instruments, United Kingdom). Body temperature was monitored using a rectal thermometer and maintained at $37^{\circ} \mathrm{C}$. Areas encompassing the distal aspect of both ischemic and nonischemic limbs were assessed for quantification of perfusion using the LDPI image processing software (v5.0). A minimum of 3 scans were obtained per limb, to minimize the variability between measurements. All results are expressed as a ratio of ischemic to contralateral nonischemic limb.

\section{Contrast enhanced ultrasound (CEU)}

At days 1 and 35 post-ischemic surgery, CEU imaging was performed using pulse inversion imaging with HDI 5000 echocardiographic system (Phillips Ultrasound, Netherlands) at transmit frequency of $3.3 \mathrm{MHz}$ (L7-4 transducer) and a mechanical index of 1.0, during intravenous (iv) infusion of lipid microbubbles $\left(1 \times 10^{7} \mathrm{~min}-1\right)$. Background images were acquired prior to microbubble infusion. Intermittent imaging was then performed by progressive prolongation of the pulsing interval (PI) from 0.2 to $20 \mathrm{~s}$, using an internal timer. Data were recorded digitally, saved to magnetic-optical disk and transferred to a computer workstation for off-line analysis. Averaged background frames were digitally subtracted from averaged contrast-enhanced frames at each PI. PI versus signal intensity (SI) data were fit to the function, $y=$ A $(1-e-\beta t)$, where $y$ is SI at the pulsing interval $t, A$ is the plateau SI which is an index of microvascular blood volume (MBV), and $\beta$ is the rate constant reflecting microvascular blood velocity. Microvascular blood flow (MBF) was calculated by the product of $A$ and $\beta$. Data was acquired from both the ischemic and control (non-ischemic) hind limb muscles below the site of iliac artery ligation.

\section{Functional assay: Treadmill}

Time to exhaustion as well as total distance on a motorized treadmill was measured to evaluate any functional changes that may be associated with changes in perfusion due to diet therapy. This test has been used in the functional evaluation of arteriogenesis and angiogenesis in animals following induction of hind limb ischemia, and improved vessel formation was found to correlate with time to exhaustion [15-18]. Time to exhaustion was defined as a characteristic change in gait. That is, a waddle of the hind quarter followed by intermittent, exaggerated hops followed by refusal to continue, or no movement in any limb for 5 seconds $[15,17,18]$. Animals were provided with 3 practice sessions prior to the final functional measurement at day 34 .

\section{Fluorescent microangiography}

Animals sacrificed at 35 days post-ligation were analyzed of blood vessel tortuosity, quantity, and length. Fluorescent microangiography was used to quantify the development of new vasculature in the proximal hind limb muscles as developed by Dr. D.J. Stewart and modified by Dr. H. Leong-Poi [19,20]. A 10\% solution of fluorescent microspheres $(2 \mu \mathrm{m}$ diameter) was mixed with a $1 \%$ solution of low melting point agarose (Sigma-Aldrich, Oakville, Ontario) and kept at 45 degrees Celsius until injection into the distal abdominal aorta immediately after the treadmill functional assay test and prior to sacrifice. Post injection of this mixture the tissue was harvested and cooled to solidify, or create a cast of, the vasculature. Thick $(200 \mu \mathrm{m})$ sections of fixed tissue were then sliced via microtome and visualized under confocal microscopy on the green fluorescent setting. These 
images were then compiled and projected in sequence to quantify the density of blood vessels via analysis by automated software (IPTK analysis software, Reindeer Graphics Inc.) which yields high resolution images of skeletal muscle vasculature in both hind limbs.

\section{Plasma biochemical analyses}

Plasma $\mathrm{B}_{6}$, determined as the semicarbazide derivative of pyridoxyl-5-phosphate, was measured using high-performance liquid chromatography (HPLC) and fluorescence detection as previously described [21]. Plasma total Hcy and Cys were quantified using ammonium 7-fluorobenzo-2-oxa-1,3-diazole-4-sulfonate by HPLC with fluorescence detection [21,22]. Vitamin B12 and folate were measured using paramagnetic particle, chemiluminescent immunoassays (Access Immunoassay Systems, Beckman Coulter Inc.) according to standardized processes at the St. Michael's Hospital Core Laboratory.

\section{Gene expression analysis}

Gene expression for eNOS and VEGF transcript levels were measured by quantitative polymerase chain reaction (qPCR) using standard techniques. Briefly, RNA was isolated from the hind limb muscle (either day 3 or day 35 tissue) using the TRIzol method, using a micropestle and sonication of the homogenized tissue, followed by the application of RNA extraction kit (Biorad Aurum RNA extraction kit). The resultant RNA was reverse transcribed using lug of RNA (cDNA synthesis kit, Biorad) at 5 minutes/25 degrees, 30 minutes/42 degrees, and 5 minutes/ 85 degrees. qPCR was performed with the resultant cDNA, and analyzed using the relative delta $\mathrm{Ct}$ value method.

\section{Circulating EPC quantification}

To calculate the EPC count in the peripheral circulation, 0.2$0.3 \mathrm{~mL}$ of blood was extracted from the tail vein at three different time points: Baseline (1 day before diet assignment), Pre-Surgery (1day pre-ligation), and Post-Surgery (2 days or 35 days post-ligation). EPCs were characterized by the presence of three endothelial cell markers: CD34, VEGFR-2, and CD133. Briefly, 100ul of blood was incubated with 1 ul of CD34, VEGFR-2 antibodies for 20 minutes at 4 degrees. It was washed with $1 \mathrm{~mL}$ of PBE buffer, and centrifuged at 380 RCF for 10 minutes. The pellet was resuspended in 100ul of PBE buffer with 1 $\mathrm{ul}$ of streptavidin (1:3 dilution) and alexa fluor 488 (1:2 dilution). Also, $10 \mathrm{uL}$ of CD133 PE-conjugated antibody was added and incubated at 4 degrees for 30 minutes. $900 \mathrm{uL}$ of lysing solution was added to lyse the RBC, and incubated for 15 minutes followed by analysis by flow cytometry (MacsQuant, Miltenyi Biotechnology).

\section{Endothelial progenitor cell isolation}

At either day 3 (early time point) or day 35 post-ligation, animals were sacrificed and EPCs were isolated using techniques previously described $[19,23]$. Briefly, tibiae and femora were isolated, and bone marrow flushed with PBS. Collected cells were plated on fibronectincoated flasks at a density of $>1 \times 10^{6}$ cells $/ \mathrm{ml}$ and maintained in Endothelial Basal Medium (EBM-2; Lonza) supplemented with EGM-2-MV SingleQuots (containing growth factors, cytokines, and supplements; Lonza) at $37^{\circ}$ Celsius and $5 \% \mathrm{CO}_{2}$. Non-adherent cells were removed after 2 days, and remaining cells were cultured for 10 days. At day 10, the EPCs were counted, and aliquoted into one of four EPC functional assays: migration assay, TUNEL assay, LECTIN assay, and tube formation assay.

\section{Migration assay}

Migration potential of EPCs was measured by using techniques previously described by Kuliszewski et al. using a Boyden chamber [19].

\section{Apoptosis assay}

The rate of apoptosis was measured by using a commercially available DeadEnd TUNEL kit (Fisher Scientific). The apoptotic rate was calculated using the following formula: (\# GFP positive)/ (\#PI positive) X $100 \%$.

\section{Differentiation assay}

Differentiation of EPCs were assayed using lectin staining. Briefly, fixed cells on 2-chambered slides were incubated in 1:250 lectin at 4 degrees overnight. After incubation, the lectin buffer was removed, and washed with PBS three times. After the final wash, the slides were mounted with Vectashield mounting medium with propidium iodide. Using 20X objective, 5 images were taken per slide and the $\%$ differentiation was calculated as following: (\# lectin positive)/ (\#PI positive) X $100 \%$.

\section{Tube formation}

The isolated EPCs were plated on matrigel-coated 6 well plates at a concentration of 200,000 cells per well and incubated at 37 degrees Celsius and $5 \% \mathrm{CO}_{2}$. At 24, 48 and 72 hours, bright field images were taken using 10X objective. Tubes formed by the cells were counted and the average length of the tubes were measured using the Image J software (ImageJ, http://imagej.nih.gov/ij/).

\section{Statistical analysis}

All experimental data is expressed as a mean \pm SD for each group unless noted otherwise. For non-parametric data, differences between groups were calculated according to Kruskal-Wallis non-parametric test (IBM SPSS ver. 21). When differences were found, Mann-Whitney test was used to discern the difference between pairwise groups with correction for multiple comparisons. For variables measured over time in the same animals (laser doppler imaging, circulating EPC numbers EPC tube length and number), repeated measures were performed with PROC MIXED in SAS using the baseline variable as a covariate [24]. Significance was determined at $\mathrm{p} \leq 0.05$.

\section{Result}

\section{Food intake and change in rodent weight}

All animals consumed comparable amounts of food and had a similar pattern of weight gain throughout the experimental period ( $\mathrm{p}=0.2$ week $5, \mathrm{p}=0.3$ week 10 , data not shown).

\section{Plasma B6, B12, folate status}

Biochemical evaluation of circulating levels of each B vitamin is found in Table 2. Early (week 5) plasma B6 concentrations in HB6 and $\mathrm{TV}$ groups were elevated in comparison with CON animals. However, this difference failed to reach significance. B12 and serum folate levels in the TV group were significantly higher than those of either the CON $(\mathrm{p}<0.01)$ or the HB6 group ( $<<0.01$ for both). There were no significant differences observed in RBC folate levels between diet groups $(\mathrm{p}=0.8)$.

At the late time point (10 weeks), plasma B6 levels were significantly elevated in HB6 $(\mathrm{p}<0.05)$ and TV $(\mathrm{p}<0.001)$ animals in comparison with $\mathrm{CON}$ animals. As expected, there was no difference in $\mathrm{B} 6$ levels between HB6 and TV groups. Vitamin B12 plasma levels were elevated in TV animals when compared to those in the HB6 group $(\mathrm{P}=0.02)$, but not when compared with $\operatorname{CON}(\mathrm{p}=0.08)$. No significant differences were seen in serum or RBC folate levels between groups $(\mathrm{p}=0.4$ for both). 
Table 2. B6, B12 and Folate concentration

\begin{tabular}{|c|c|c|c|c|c|c|c|c|}
\hline & \multicolumn{2}{|c|}{ B6 (nmol/L) } & \multicolumn{2}{|c|}{ B12 (pmol/L) } & \multicolumn{2}{|c|}{ Serum Folate (nmol/L) } & \multicolumn{2}{|c|}{ RBC Folate $(\mu \mathrm{mol} / \mathrm{L})$} \\
\hline & Week 5 & Week 10 & Week 5 & Week 10 & Week 5 & Week 10 & Week 5 & Week 10 \\
\hline $\mathrm{CON}^{1}$ & $736(608,867)(12)$ & $572(424,572)(10)$ & $989(906,1047)(16)$ & $799(752,847)(2)$ & $104(77,128)(19)$ & $95(84,107)(2)$ & $1149(1006,1482)(17)$ & $787(758,817)(2)$ \\
\hline HB6 & $814(727,1041)(9)$ & $1098(695,1257)^{\dagger}(10)$ & $936(870,1074)(15)$ & $838(787,928)(6)$ & $97(91,104)(17)$ & $88(84,91)(6)$ & $1071(1071,1443)(16)$ & $840(757,947)(6)$ \\
\hline TV & $918(740,1057)(9)$ & $1204(839,1401)^{\ddagger}(10)$ & $1190(1056,1515)^{*, \#}(17)$ & $1281(1267,2207)^{\&}(4)$ & $126(116,137)^{*, \#}(19)$ & $151(80,216)(4)$ & $1135(906,1503)(18)$ & $1055(903,1064)(4)$ \\
\hline
\end{tabular}

$* \mathrm{p}<0.05$ vs. $\mathrm{CON}, \uparrow \mathrm{p}<0.01$ vs. $\mathrm{CON}, \mathrm{t}<<0.001$ vs. $\mathrm{CON}, \& \mathrm{p}=0.02$ vs. $\mathrm{HB} 6, \# \mathrm{p}<0.01$ vs. HB6.

(n) indicates sample size.

Values are expressed as median $\left(25^{\text {th }}, 75^{\text {th }}\right)$.

CON: Control

HB6: High vitamin B6

TV: Tri-vitamin Group

\section{Homocysteine and cysteine status}

At the time of sacrifice, no significant differences in plasma cysteine levels between diet treatment groups were observed $(p=0.6$; Table 3). Circulating levels of HCy were reduced in animals receiving tri-vitamin supplementation, however, differences between groups did not reach statistical significance $(\mathrm{p}=0.07)$.

\section{Blood flow by contrast enhanced ultrasound (CEU)}

CEU data was expressed as a ratio of blood flow between the ischemic limb compared to the non-ischemic limb (Figure 2). Both CON and HB6 exhibited a significant increase in blood flow from day 1 to day 35 post-ischemia. The TV group showed a modest yet nonsignificant increase within the same timeframe. Day 1 post surgery all treatment groups had a similar volume of blood flow, however, at day 35 the CON animals had a significantly higher blood flow in the ischemic limb in comparison to both HB6 $(\mathrm{p}<0.05)$ and TV animals $(\mathrm{p}<0.01)$.

\section{Blood flow by laser doppler perfusion imaging (LDPI)}

Ligation predominantly affects perfusion of the foot. Blood flow measured by laser Doppler imaging in the feet of experimental animals at day 1, 7, 14 and 35 revealed a significant increase in perfusion with time $(\mathrm{p}<0.002)$. There was also an independent effect of dietary treatment $(\mathrm{p}=0.04)$. Graphically, animals had similar blood flow changes over time with the blood flow measurements being similar between the control and HB6 animals. Reduced perfusion in the foot of tri-vitamin group at all time points. There was no significant time by diet treatment interaction $(\mathrm{p}=0.9)$ (Table 4).

\section{Expression of VEGF and eNOS}

Gene expression data is summarized in Figure 3. Gene expression of VEGF and eNOS were not significantly altered by the different dietary treatments at the early ( 5 week) time point $(\mathrm{p} \geq 0.6)$. However, at the 10week time point, both VEGF and eNOS expression was elevated in the CON group $(135 \pm 38 \%$ and $233 \pm 88 \%)$ in comparison with the HB6 (107 $\pm 24 \%$ and $109 \pm 24 \%, \mathrm{p}<0.05$ and 0.01$)$ and TV $(78 \pm$ $31 \%$ and $84 \pm 32 \%, \mathrm{p}<0.01$ and 0.01$)$. In addition, both VEGF and eNOS expression were found to be significantly higher in the HB6 group when compared to the TV group at the 10week time point $(\mathrm{p}<0.05$ respectively). While there was no significant change in VEGF expression within groups over time, eNOS expression was significantly reduced in the HB6 $(\mathrm{p}<0.05)$ and TV groups $(\mathrm{p}<0.01)$.

\section{Circulating EPC quantification}

Repeated measures analysis of the number of circulating EPCs measured at baseline, pre-surgery, and 2 days post-surgery (early time point) revealed no significant effect of diet treatment $(\mathrm{p}=0.5)$. However, there was a significant effect of time $(\mathrm{p}=0.02)$. There was no interaction
Table 3. Plasma concentration of Cysteine and Homocysteine

\begin{tabular}{|c|c|c|}
\hline & Cysteine $(\boldsymbol{\mu m o l} / \mathbf{L})$ & Homocysteine $(\boldsymbol{\mu m o l} / \mathbf{L})$ \\
\hline CON $^{1}$ & $191 \pm 34(16)$ & $5.5 \pm 1.5(16)$ \\
\hline HB6 & $201 \pm 19(13)$ & $5.4 \pm 0.9(13)$ \\
\hline TV & $190 \pm 32(15)$ & $4.5 \pm 1 \quad(15)$ \\
\hline
\end{tabular}

(n) indicates sample size.

Values are expressed as mean $\pm \mathrm{SD}$

CON: Control

HB6: High vitamin B6

TV: Tri-vitamin Group

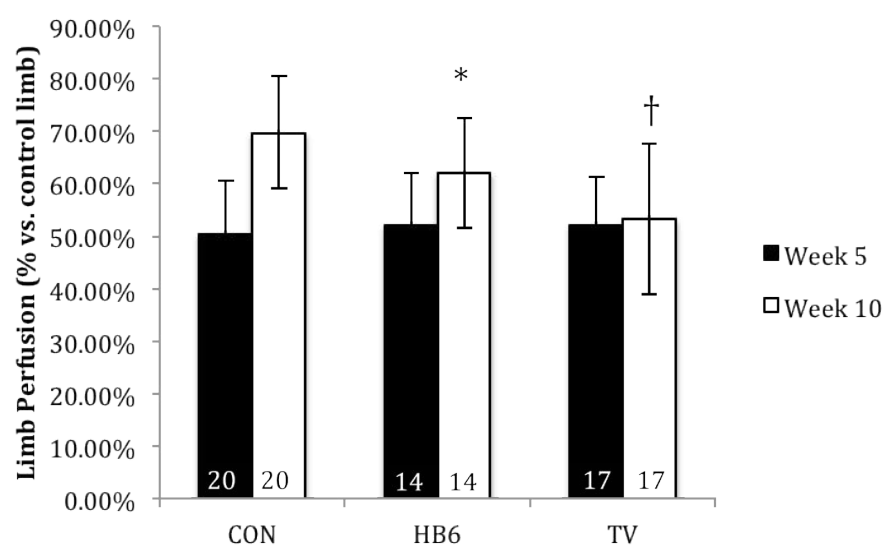

Figure 2. Effect of B-vitamins on hind limb perfusion. Perfusion percent normalized to the contralateral normal limb. Numbers inside bars represent sample size. ${ }^{*} \mathrm{p}<0.05$ vs. CON $\dagger \mathrm{p}<0.01$ vs. CON. Values are represented as mean \pm SD.

between time and treatment $(\mathrm{p}=0.3)$. At 35 days post-ligation, the number of circulating EPCs was reduced in both HB6 and TV animals (Figure 4). This reduction was significant for TV animals ( $\mathrm{p}=0.01$ ), but not HB6 animals $(\mathrm{p}=0.2)$.

\section{Bone marrow-derived EPC tube number and length}

EPCs isolated from treated animals failed to show a time dependent change in tube number $(\mathrm{p}=0.2)$ over the 72 hours. However, a significant effect of treatment was observed $(p=0.02)$. Control animals demonstrated an increased number of tubes formed over the 72 hours period in comparison with either vitamin B6 alone or tri-vitamin fed animals (Table 5). Tube length also suggested a significant effect of treatment group on EPC tube length $(\mathrm{p}=0.03)$ but no effect of time $(p=1.0)$. Control animals demonstrated a longer tube length in comparison with either the $\mathrm{B} 6$ or tri-vitamin treated animals. There was, however, a significant interaction of time and treatment $(\mathrm{p}=0.04)$ (Table 5).

\section{Migration potential of bone marrow-derived EPCs}

For EPCs isolated at week 5, migration was significantly enhanced with HB6 supplementation $(\mathrm{p}=0.01)$ when compared with $\mathrm{CON}$ 

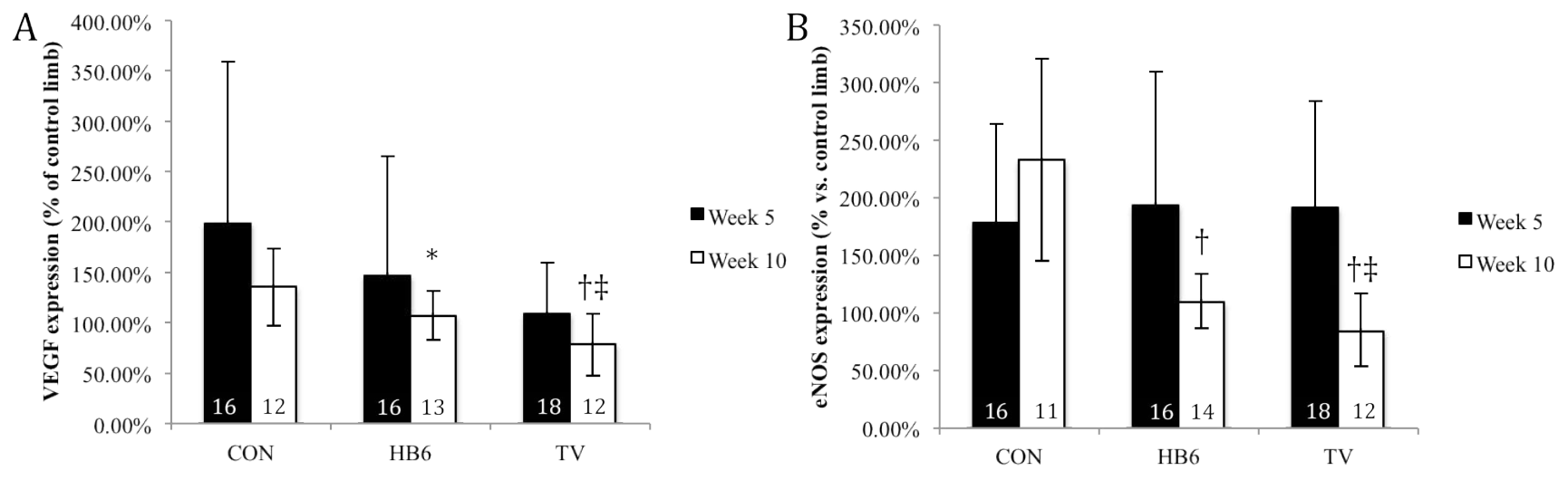

Figure 3. Effect of B-vitamins on VEGF and eNOS expression. Expression percent normalized to the contralateral normal limb. Numbers inside bars represent sample size. *p $<0.05$ vs. $\mathrm{CON}, \pitchfork \mathrm{p}<0.001$ vs. CON, $₫ \mathrm{p}<0.05$ vs. HB6. Values are represented as mean $\pm \mathrm{SD}$.

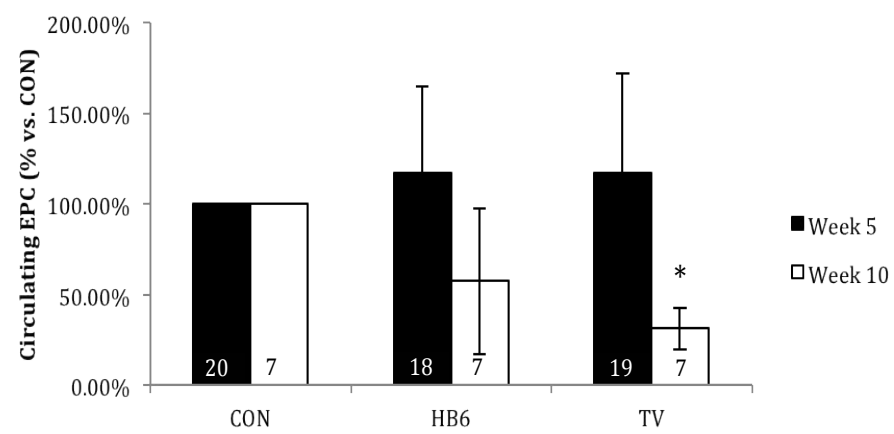

Figure 4. Effect of B-vitamin(s) on circulating EPCs in vivo. All groups are normalized to CON. Numbers inside bars represent sample size. ${ }^{*} \mathrm{p}<0.05$ vs $\mathrm{CON}$ at week 10 . Values are represented as mean $\pm \mathrm{SD}$.

Table 4. Laser Doppler Perfusion Imaging of the Foot

\begin{tabular}{|c|c|c|c|c|} 
& Day 1 & Day 7 & Day 14 & Day 35 \\
\hline CON $^{1}$ & $46 \pm 15 \%$ & $55 \pm 14 \%(17)$ & $65 \pm 20 \%(18)$ & $75 \pm 20 \%(20)$ \\
\hline HB6 & $46 \pm 21 \%$ & $67 \pm 18 \%(10)$ & $71 \pm 17 \%(12)$ & $78 \pm 18 \%(18)$ \\
\hline TV & $45 \pm 16 \%$ & $54 \pm 18 \%(14)$ & $62 \pm 17 \%(16)$ & $69 \pm 16 \%(13)$ \\
\hline
\end{tabular}

Percent perfusion is expressed as percent of contralateral foot (internal control). (n) indicates sample size.

Values are expressed as mean $\pm \mathrm{SD}$

EPC: Endothelial Progenitor Cell

CON: Control

HB6: High vitamin B6

TV: Tri-vitamin Group

while the TV group was not significantly different from $\operatorname{CON}(\mathrm{p}=0.3)$. However, EPCs isolated at week 10 did not yield significant differences in migration potential relative to CON. Migration potential for HB6 dropped significantly from week 5 to week $10(\mathrm{p}=0.02)$, while the changes seen in CON and TV within the same time frame were not significant ( $\mathrm{p}=0.9$ and 0.1 , respectively)

\section{Differentiation of bone marrow-derived EPCs}

Effect of diet on EPC differentiation is summarized in Figure 5A. There was no effect of diet treatment on EPC differentiation at either the early ( 5 week) or late ( 10 week) time point when compared with $\operatorname{CON}(\mathrm{p}=0.5$ early and $\mathrm{p}=0.6$ late) .

\section{EPC apoptosis}

Effect of diet on apoptosis is summarized in Figure 5B. There was no effect of diet treatment on EPC apoptosis between treatment groups at either the early or late time point ( $\mathrm{p}=0.4$ and $\mathrm{p}=0.9$ respectively).

\section{Functional effect by exercise capacity test}

Functional capacity, as measured by the time and distance rodents traveled on a treadmill was not different at day 35 between diet treatment groups (data not shown).

\section{Fluorescent microangiography}

There were no significant differences in either tortuosity $(\mathrm{p}=0.5)$, quantity $(\mathrm{p}=0.4)$, total length $(\mathrm{p}=0.2)$, or mean length $(\mathrm{p}=0.3)$ of the hind limb blood vessels as measured by FMA between treatment groups (data not shown).

\section{Discussion}

Homocysteine's putative role in CVD has been well studied in the last few decades. It is generally accepted that Hcy level is a powerful risk factor of CVD despite a lack of consensus on whether lowering Hcy levels will lead to improvements in CVD patients [25-31]. Therefore, consumers and clinicians may view B vitamin supplementation as a relatively harmless adjunctive therapy with potential health benefits including both the restoration of circulating levels as well as a reduced CVD risk. The current data suggests that consumption of $B$ vitamins can negatively impact the recovery of perfusion following an ischemic event. Rodents provided with a diet enriched with B6 demonstrated an impaired recovery of perfusion in the ischemic limb together with a reduction in circulating endothelial progenitor cells and mediators known to stimulate the angiogenic response (VEGF and eNOS). The impairment in this adaptive response was enhanced in animals receiving B6 in combination with high levels of B12 and folic acid. While no negative impact of supplementation was apparent immediately post-induction of ischemia (early time point), impaired perfusion was observed following five weeks of recovery. These findings support the hypothesis that B vitamin supplementation results in an impairment of the adaptive responses to an ischemic event leading to impaired recovery.

The hypothesis that $\mathrm{B}$ vitamins exert an anti-angiogenic response is rooted in both epidemiologic and laboratory studies. Epidemiologic and case control studies have found evidence of an inverse relationship between B6 consumption and cancer risk [32-38]. In vitro studies by Matsubara etaldemonstrated that rat aortic rings cultured in the presence of increasing concentrations of vitamin B6 (PLP) demonstrated a dosewise reduction in vessel outgrowth [6]. Furthermore, mice treated with PLP demonstrated a reduction in both the number and size of colon tumors developed following azoxymethane treatment in comparison with non-supplemented animals [4,5]. These protective findings were, 

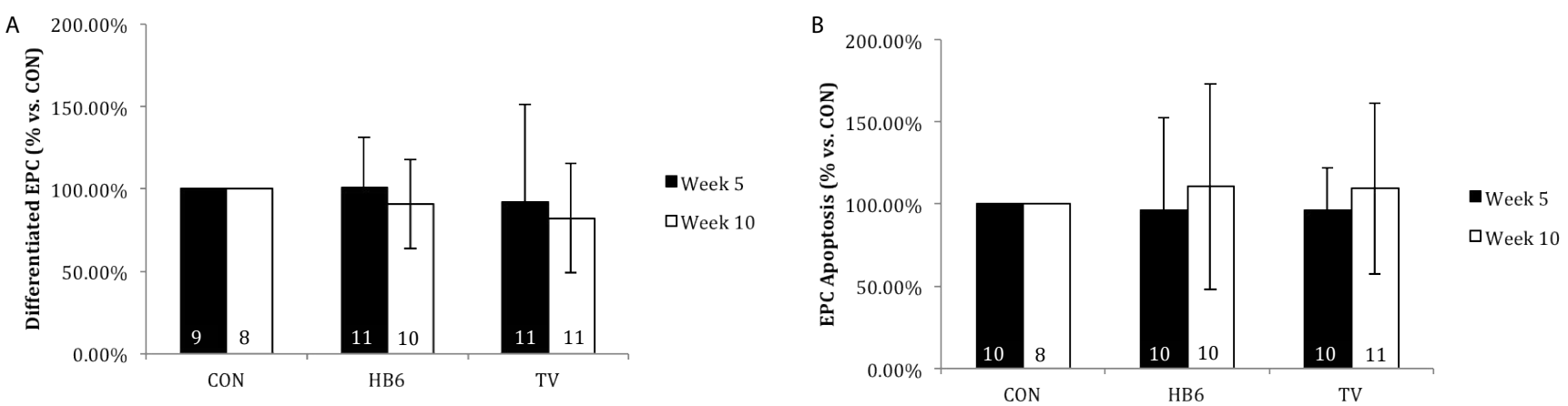

Figure 5. Effect of B-vitamins on EPC differentiation (A) and apoptosis (B). All groups are normalized to CON. Numbers inside bars represent sample size. Values are represented as mean $\pm \mathrm{SD}$.

Table 5. Quantification of the number and length of $\mathrm{EPC}^{4}$ tubes formed at 24,48 and 72 hours in culture

\begin{tabular}{|c|c|c|c|c|c|c|c|c|c|}
\hline & \multicolumn{3}{|c|}{24 hours } & \multicolumn{3}{|c|}{48 hours } & \multicolumn{3}{|c|}{72 hours } \\
\hline & CON & HB6 & TV & CON & HB6 & TV & CON & HB6 & TV \\
\hline Tube Number & $31.7 \pm 17.4(21)$ & $34.1 \pm 24.0(26)$ & $32.3 \pm 20.8(27)$ & $41.7 \pm 21.1(21)$ & $37.8 \pm 24.7(26)$ & $37.2 \pm 21.8(27)$ & $47.3 \pm 25.2(21)$ & $39.3 \pm 23.1(26)$ & $41.3 \pm 22.8(27)$ \\
\hline Tube Length & $71.2 \pm 16.9(21)$ & $65.9 \pm 26.7(26)$ & $71.5 \pm 29.2(27)$ & $72.9 \pm 17.9(21)$ & $65.8 \pm 20.8(26)$ & $70.2 \pm 23.6(27)$ & $72.6 \pm 17.4(21)$ & $70.9 \pm 22.9(26)$ & $65.3 \pm 21.1$ \\
\hline
\end{tabular}

(n) indicates sample size.

Values are expressed as mean $\pm \mathrm{SD}$

at least in part, found to be related to a reduction in oxidative stress, cell proliferation, nitric oxide production and angiogenesis associated with B vitamin supplementation and resulted in a reduction in tumor growth [5,39].

In response to an ischemic injury, EPCs migrate to the site of injury where they proliferate and are incorporated into new blood vessels using the process of angiogenesis. EPCs may accelerate endothelization at the site of vascular damage providing a repair mechanism for reendothelization and neoangiogenesis [40]. Therefore, improvements in perfusion may be related to either the process of arteriogenesis (remodeling of previously formed channels into larger ones) or to angiogenesis (the growth of new collateral arterioles and collateral arteries) $[41,42]$. In this study, improvement in hind limb perfusion observed with contrast enhanced ultrasound between groups may be related to an increased number or length of developed blood vessels. In addition, the number of circulating EPCs at week 10 ( 35 days postsurgery) was significantly reduced in the TV group. EPC number was also shown to be decreased in HB6 group, but the differences did not reach significance. A reduction, in circulating EPC levels could contribute to a reduction in the angiogenic response in these animals. It is important to note that circulating EPCs were not affected by diet treatment alone as indicated by pre-surgical quantitation. However, while in vitro work did suggest some potential differences in the number and length of developed tubes, we did not observe any differences in EPC differentiation, migration or apoptosis between dietary treatment groups making conclusions regarding the impact of $B$ vitamin supplementation on the maturation of EPCs into endothelial cells or their premature death difficult. Our findings are supported by the work of Komatsu and colleagues who demonstrated that $\mathrm{B}$ vitamins, specifically B6, did not affect cellular apoptosis [4]. Finally, we also did not observe an increase in the number of blood vessels between treatment groups using fluorescent microangiography, it is possible that either arteriogenesis (the increase in caliber of preexistent vessels), or improved vessel repair, occurred in the controls; resulting in improved perfusion in this group.

Angiogenesis is a complex process with multiple pathways. One of the most potent growth factors for the angiogenic response is
VEGF which acts as a chemotactic factor in the recruitment of EPCs promoting proliferation and angiogenesis [42-44]. In addition, eNOS also plays a critical role in the angiogenic response. A reduction in eNOS expression is linked with reduced proliferation, migration and adhesion of EPCs and increased apoptosis [45]. In addition, eNOS increases the production of nitric oxide, which can induce angiogenic responses in vivo and is further induced by VEGF expression [4648]. Reduced nitric oxide production is associated with an impaired angiogenic response $[49,50]$.

Both VEGF and eNOS are up-regulated in situations of increased oxidative stress [51,52]. Therefore, one hypothesis for the observed effects of B vitamins in this study may be through the modulation of oxidative stress following the induction of ischemia. Each of the supplemented vitamins has been shown in both animal and human studies to reduce oxidative stress in vivo. Supplementation of B vitamins in patients following an acute ischemic stroke demonstrated in increase in total antioxidant capacity together with a reduction in malondialdehyde (MDA) and C-reactive protein (CRP) [53]. As part of Boston Puerto Rican Health Study, Shen et al. demonstrated showed a strong dose dependent relationship between PLP and CRP. Lower PLP levels were linked with inflammation and higher oxidative stress in older Peurto Rican Adults [33]. B6 is a strong quencher of singlet oxygen and can reduce lipid peroxidation [54-56]. B6 deficiency has been linked to increased lipid peroxidation and activation of glutathione dependent enzyme [56]. In rodents, B6 deficiency led to decreased catalase and SOD activity as well as a reduction in the GSH/ GSSG ratio with increased levels of MDA [33,57]. Reduced GSG/GSSG ratio suggests decreased antioxidant protection and increased reactive oxygen species (ROS). Folate has also been shown to act as a free radical scavenger [58]. Nakano [59] demonstrated a direct antioxidant effect of folate on LDL oxidation which was independent of HCY lowering. In addition, folate can stabilize or re-couple eNOS resulting in a reduction in nitric oxide production [60]. Vitamin B6 supplementation alone led to the down-regulation of both VEGF and eNOS expression, an effect that was enhanced with the addition of B12 and folate. Therefore, one hypothesis for the observed findings is a modulation of post ischemic oxidative stress resulting in an environment less likely to promote angiogenesis through the reduction in VEGF and eNOS expression 
resulting in reduced stimulation for EPC release from the bone marrow, impaired arteriogenesis and subsequently a reduction in the recovery of perfusion.

There are a number of limitations in the study. The use of fluorescent microangiography as a measure of determining the extent of angiogenesis is not robust. Difficulty in the quantification and visualization of vessels may have resulted in the lack of difference observed between groups and therefore the lack of support for an angiogenic response. In vitro work (migration, differentiation and apoptosis) may have produced weak or inconclusive findings due to the lack of B vitamins within the media. EPCs isolated from bone marrow may have lost or have a reduced inhibitory effect of $\mathrm{B}$ vitamin supplementation in vivo when plated and grown in culture, thereby negating any potential differences between diet groups. The completion of multiple studies in different tissues also limited the sample size available for each study reducing our ability to do more detailed comparisons between groups. In addition, we were not able to discern any difference between groups in the functional outcome of increased perfusion via the treadmill technique. The absence of significance in the differences in exercise capacity measured by the total distance or total time on the treadmill may be due to either: the lack of discerning power of the test in determining the subtle differences in the functional capacity or; the observed increase in perfusion may not translate to significant increase in functional improvements. A final limitation, is that we were did not measure any markers of oxidative stress. Further studies are required to clarify the impact of B vitamin supplementation on oxidative stress as a mechanism for modulating the angiogenic response.

In conclusion, this study suggests potentially adverse effects associated with B6 supplementation on the recovery of tissue perfusion following an ischemic injury. This effect may be, in part, due to the effect of B6 on EPC chemotactic function as suggested by the altered VEGF and eNOS gene expression. These findings were enhanced by the addition of folate and B12. Modulation of the expression of these angiogenic genes may be the result of reduced oxidative stress as a result of B vitamin supplementation. While alterations in the angiogenic response may be beneficial in the context of tumor growth and development, a blunting of this adaptive response following an ischemic injury may be detrimental as seen in the NORVIT trial. These finding suggest that caution should be observed when considering $B$ vitamin supplementation in patients with underlying ischemia.

\section{Acknowledgement}

Statistical Analysis in SASS was provided by Dr. Aiala Barr.

MK, HLP, MK designed research; MK, PL wrote paper; MK, PL analyzed the data; MK had primary responsibility for the final content; JH, SD, NY, LH, PL, CL, MK, PAN, MA conducted research; HLP provided essential reagents

All authors have read and reviewed the final manuscript.

\section{References}

1. Toole JF, Malinow MR, Chambless LE, Spence JD, Pettigrew LC, et al. (2004) Lowering homocysteine in patients with ischemic stroke to prevent recurrent stroke, myocardial infarction, and death: the Vitamin Intervention for Stroke Prevention (VISP) randomized controlled trial. JAMA 291: 565-575. [Crossref]

2. Lonn E, Yusuf S, Arnold MJ, Sheridan P, Pogue J, et al. (2006) Homocysteine lowering with folic acid and B vitamins in vascular disease. N Engl J Med 354: 1567-1577.

3. Bonaa KH, Njolstad I, Ueland PM, Schirmer H, Tverdal A, et al. (2006) Homocysteine lowering and cardiovascular events after acute myocardial infarction. $N$ Engl J Med 354: 1578-1588. [Crossref]
4. Komatsu SI, Watanabe H, Oka T, Tsuge H, Nii H, et al. (2001) Vitamin B-6supplemented diets compared with a low vitamin B-6 diet suppress azoxymethaneinduced colon tumorigenesis in mice by reducing cell proliferation. J Nutr 131: 22042207. [Crossref]

5. Komatsu S, Watanabe H, Oka T, Tsuge H, Kat N (2002) Dietary vitamin B6 suppresses colon tumorigenesis, 8-hydroxyguanosine, 4-hydroxynonenal, and inducible nitric oxide synthase protein in azoxymethane-treated mice. J Nutr Sci Vitaminol (Tokyo) 48: 65-68. [Crossref]

6. Matsubara K, Mori M, Matsuura Y, Kato N (2001) Pyridoxal 5'-phosphate and pyridoxal inhibit angiogenesis in serum-free rat aortic ring assay. Int $\mathrm{J} \mathrm{Mol} \mathrm{Med} \mathrm{8:}$ 505-508. [Crossref]

7. Hanninen SA, Darling PB, Sole MJ, Barr A, Keith ME (2006) The prevalence of thiamin deficiency in hospitalized patients with congestive heart failure. $J$ Am Coll Cardiol 47: 354-361. [Crossref]

8. Keith ME, Walsh N, Darling P, Hanninen S, Barr A, et al. (2009) B-vitamin deficiency in hospitalized patients with heart failure. J Am Diet Assoc 109: 1406-1410. [Crossref]

9. Reeves PG, Nielsen FH, Fahey Jr GC (1993) AIN-93 Purified Diets for Laboratory Rodents: Final Report of the American Institute of Nutrition Ad Hoc Writing Committee on the Reformulation of the AIN-76A Rodent Diet. J Nutr 123: 1939-1951. [Crossref]

10. National Research Council (1995) Nutrient requirements of laboratory animals Washington,DC: National Academy Press. [Crossref]

11. Mangoni AA, Sherwood RA, Asonganyi B, Swift CG, Thomas S, et al. (2005) Shortterm oral folic acid supplementation enhances endothelial function in patients with type 2 diabetes. Am J Hypertens 18: 220-226. [Crossref]

12. Tawakol A, Migrino RQ, Aziz KS, Waitkowska J, Holmvang G, et al. (2005) Highdose folic acid acutely improves coronary vasodilator function in patients with coronary artery disease. J Am Coll Cardiol 45: 1580-1584. [Crossref]

13. Woodman RJ, Celermajer DE, Thompson PL, Hung J (2004) Folic acid does not improve endothelial function in healthy hyperhomocysteinaemic subjects. Clin Sci (Lond) 106: 353-358.

14. Leong-Poi H, Christiansen J, Heppner P, Lewis CW, Klibanov AL, et al. (2005) Assessment of endogenous and therapeutic arteriogenesis by contrast ultrasound molecular imaging of integrin expression. Circulation 111: 3248-3254. [Crossref]

15. Ikenaga S, Hamano K, Nishida M, Kobayashi T, Li TS, et al. (2001) Autologous bone marrow implantation induced angiogenesis and improved deteriorated exercise capacity in a rat ischemic hindlimb model. J Surg Res 96: 277-283. [Crossref]

16. Yoshida J, Ohmori K, Takeuchi H, Shinomiya K, Namba T, et al. (2005) Treatmen of ischemic limbs based on local recruitment of vascular endothelial growth factorproducing inflammatory cells with ultrasonic microbubble destruction. $J$ Am Coll Cardiol 46: 899-905.

17. Orito K, Kishi M, Fujiki H, Nakazawa T, Imaizumi T, et al. (2004) A method for evaluating drug effects on intermittent claudication using a treadmill in rats with unilateral hindlimb artery occlusion. J Pharmacol Toxicol Methods 49: 25-29.

18. Lloyd PG, Yang HT, Terjung RL (2001) Arteriogenesis and angiogenesis in rat ischemic hindlimb: role of nitric oxide. Am J Physiol Heart Circ Physiol 281: H25282538. [Crossref]

19. Kuliszewski MA, Zucco L, Faughnan M, Swan L, Therrien J, et al. (2003) Phenotypic characterization of circulating endothelial progenitor cells in genetically-determined vascular disorders. Circulation 108: 2392.

20. Dutly AE, Kugathasan L, Trogadis JE, Keshavjee SH, Stewart DJ, et al. (2006) Fluorescent microangiography (FMA): an improved tool to visualize the pulmonary vasculature. Lab Invest 86: 409-416. [Crossref]

21. Mayengbam S, Raposo S, Aliani M, House JD (2015) Oral exposure to the antipyridoxine compound 1-amino D-proline further perturbs homocysteine metabolism through the transsulphuration pathway in moderately vitamin B6-deficient rats. $J$ Nutr Biochem 26: 241-249. [Crossref]

22. Zhang Z, Kebreab E, Jing M, Rodriguez-Lecompte JC, Kuehn R, et al. (2009) Impairments in pyridoxine-dependent sulphur amino acid metabolism are highly sensitive to the degree of vitamin B6 deficiency and repletion in the pig. Animal 3 : 826-837. [Crossref]

23. Verma S, Kuliszewski MA, Li SH, Szmitko PE, Zucco L, et al. (2004) C-reactive protein attenuates endothelial progenitor cell survival, differentiation, and function: further evidence of a mechanistic link between C-reactive protein and cardiovascular disease. Circulation 109: 2058-2067. [Crossref] 
24. Wang Z, Goonewardene LA (2004) The use of MIXED models in the analysis of animal experiments with repeated measures data. Can J Anim Sci 84: 1-11.

25. Verhoef P, Kok FJ, Kruyssen DA, Schouten EG, Witteman JC, et al. (1997) Plasma total homocysteine, B vitamins, and risk of coronary atherosclerosis. Arterioscler Thromb Vasc Biol 17: 989-995. [Crossref]

26. Boushey CJ, Beresford SA, Omenn GS, Motulsky AG (1995) A quantitative assessment of plasma homocysteine as a risk factor for vascular disease. Probable benefits of increasing folic acid intakes. JAMA 274: 1049-1057. [Crossref]

27. van den BM, Stehouwer CD, Bierdrager E, Rauwerda JA (1996) Plasma homocysteine and severity of atherosclerosis in young patients with lower-limb atherosclerotic disease. Arterioscler Thromb Vasc Biol 16: 165-171. [Crossref]

28. Graham IM, Daly LE, Refsum HM, Robinson K, Brattstrom LE, et al. (1997) Plasma homocysteine as a risk factor for vascular disease. The European Concerted Action Project. JAMA 277: 1775-1781. [Crossref]

29. Chasan-Taber L, Selhub J, Rosenberg IH, Malinow MR, Terry P, et al. (1996) A prospective study of folate and vitamin B6 and risk of myocardial infarction in US physicians. J Am Coll Nutr 15: 136-143. [Crossref]

30. Evans RW, Shaten BJ, Hempel JD, Cutler JA, Kuller LH (1997) Homocyst(e)ine and risk of cardiovascular disease in the Multiple Risk Factor Intervention Trial. Arterioscler Thromb Vasc Biol 17: 1947-1953. [Crossref]

31. Folsom AR, Nieto FJ, McGovern PG, Tsai MY, Malinow MR, et al. (1998) Prospective study of coronary heart disease incidence in relation to fasting total homocysteine, related genetic polymorphisms, and B vitamins: The Atherosclerosis Risk in Communities (ARIC) study. Circulation 98: 204-210. [Crossref]

32. Slattery ML, Potter JD, Coates A, Ma KN, Berry TD, et al. (1997) Plant foods and colon cancer: an assessment of specific foods and their related nutrients. Cancer Causes Control 8: 575-590. [Crossref]

33. Shen J, Lai C-Q, Mattei J, Tucker KL (2010) Association of vitamin B-6 status with inflammation, oxidative stress and chronic inflammatory conditions: the Boston Puerto Rican Health Study. Am J Clin Nutr 91: 337-342. [Crossref]

34. Larsson SC, Orsini N, Wolk A (2010) Vitamin B6 and risk of colorectal cancer: a metaanalysis of prospective studies. JAMA 303: 1077-1083. [Crossref]

35. Lee JE, Li H, Giovannucci E, Lee IM, Selhub J, et al. (2009) Prospective study of plasma vitamin B6 and risk of colorectal cancer in men. Cancer Epidemiol Biomarkers Prev 18: 1197-1202. [Crossref]

36. Eussen SJ, Vollset SE, Hustad S, Midttun Ø, Meyer K, et al. (2010) Plasma vitamins $\mathrm{B} 2, \mathrm{~B} 6$, and B12, and related genetic variants as predictors of colorectal cancer risk. Cancer Epidemiol Biomarkers Prev 19: 2549-2561. [Crossref]

37. Le Marchand L, Wang H, Selhub J, Vogt TM, Yokochi L, et al. (2011) Association of plasma vitamin B6 with risk of colorectal adenoma in a multiethnic case-control study. Cancer Causes Control 22:929-936. [Crossref]

38. Wei EK, Giovannucci E, Selhub J, Fuchs CS, Hankinson SE, et al. (2005) Plasma vitamin B6 and the risk of colorectal cancer and adenoma in women. $J$ Natl Cancer Inst 97: 684-692. [Crossref]

39. Komatsu S, Yanaka N, Matsubara K, Kato N (2003) Antitumor effect of vitamin B6 and its mechanisms. Biochim Biophys Acta 1647: 127-130. [Crossref]

40. Jung C, Rafnsson A, Shemyakin A, Bohm F, Pernow J (2010) Different subpopulations of endothelial progenitor cells and circulating apoptotic progenitor cells in patients with vascular disease and diabetes. Int J Cardiol 143: 368-372. [Crossref]

41. Angoulvant D, Fazel S, Li Ren-Ke (2004) Neovascularization derived from transplantation in ischemic myocardium. Mol Cell Biochem 264: 133-142. [Crossref]
42. Fam NP, Verma S, Kutryk M, Stewart DJ (2003) Clinician guide to angiogenesis. Circulation 108: 2613-2618. [Crossref]

43. Bergers G, Benjamin LE (2003) Tumorigenesis and the angiogenic switch. Nat Rev Cancer 3: 401-410. [Crossref]

44. Pepper MS, Ferrara N, Orci L, Montesano R (1992) Potent synergism between vascular endothelial growth factor and basic fibroblast growth factor in the induction of angiogenesis in vitro. Biochem Biophys Res Commun 189: 824-831. [Crossref]

45. Chen J, Jin J, Song M, Dong H, Zhao G, et al. (2012) C-reactive protein down-regulates endothelial nitric oxide synthase expression and promotes apoptosis in endothelial progenitor cells through receptor for advanced glycation end products. Gene 496: 128135. [Crossref]

46. Murohara T, Asahara T, Silver M, Bauters C, Masuda H, et al. (1998) Nitric oxide synthase modulates angiogenesis in response to tissue ischemia. J Clin Invest 10: 25672578. [Crossref]

47. Bouloumie A, Schini-Kerth VB, Busse R (1999) Vascular endothelial growth factor up-regulates nitric oxide synthase expression in endothelial cells. Cardiovasc Res 41:773-780. [Crossref]

48. Hood JD, Meininger CJ, Ziche M, Granger HJ (1998) VEGF upregulates ecNOS message, protein, and NO production in human endothelial cells. Am J Physiol 274(3 Pt 2): H1054-H1058. [Crossref]

49. Matsunaga T, Weihrauch DW, Moniz MC, Tessmer J, Warltier DC, et al. (2002) Angiostatin inhibits coronary angiogenesis during impaired production of nitric oxide. Circulation 105: 2185-2191. [Crossref]

50. Verma S, Wang CH, Li SH, Aaron SD,Paul WMF et al. (2002) A self-fulfilling prophecy: $\mathrm{C}$ reactive protein attenuates nitric oxide production and inhibits angiogenesis. Circulation 105: 735-741.

51. Maulik N, Das DK (2002) Redox signaling in vascular angiogenesis. Free Radic Biol Med 33: 1047-1060. [Crossref]

52. Ding H, Aljofan M, Triggle CR (2007) Oxidative stress and increased eNOS and NADPH oxidase expression in mouse microvessel endothelial cells. J Cell Physiol 212: 682-689. [Crossref]

53. Ullegaddi R, Powers HJ, Gariballa SE (2006) Antioxidant supplementation with or without B- group vitamins after acute ischemic stroke: a randomized controlled trial. JPEN J Parenter Enteral Nutr 30: 108-114. [Crossref]

54. Jain SK, Lim G (2001) Pyridoxine and pyridoxamine inhibits superoxide radicals and prevents lipid peroxidation, protein glycosylation and $(\mathrm{Na}++\mathrm{K}+)$-ATP ase activity reduction in high glucose-treated human erythrocytes. Free Radic Biol Med 30: 232237. [Crossref]

55. Kannan K, Jain SK (2004) Effect of vitamin B6 on oxygen radicals, mitochondria membrane potential, and lipid peroxidation in $\mathrm{H} 202$ treated U937 monocytes. Free Radic Biol Med 36:423-428. [Crossref]

56. Cabrini L, Bergami R, Fiorentini D, Marchetti M, Landi L, et al. (1998) Vitamin B6 deficiency affects antioxidant defences in rat liver and heart. Biochem Mol Biol Int 46: 689-697. [Crossref]

57. Förstermann U, Münzel T (2006) Endothelial nitric oxide synthase in vascular disease: from marvel to menace. Circulation 113: 1708-1714. [Crossref]

58. Wilmink HW, Stroes ES, Erkelens WD et al. (2000) Influence of folic acid on postprandial endothelial dysfunction. Arterioscler, Thromb, Vasc. Biol 20:185-188.

59. Nakano E, Higgins JA, Powers HJ (2001) Folate protects against oxidative modification of human LDL. Br J Nutr 86: 637-639. [Crossref]

60. Verhaar MC, Stroes E, Rabelink TJ (2002) Folates and cardiovascular disease Arterioscler Thromb Vasc Biol 22: 6-13. [Crossref]

Copyright: (C2017 Lee PJ. This is an open-access article distributed under the terms of the Creative Commons Attribution License, which permits unrestricted use, distribution, and reproduction in any medium, provided the original author and source are credited. 